Se prémunir contre les préjugés ouvriers.

L'économie politique des machines entre l'Angleterre et la France(1800-1850)

Preventing labour prejudices : the political economy of

machinery between England and France (1800-1850)

\title{
François Jarrige
}

\section{OpenEdition}

Journals

\section{Édition électronique}

URL : http://journals.openedition.org/dht/1518

DOI : $10.4000 /$ dht. 1518

ISSN : $1775-4194$

Éditeur :

Centre d'histoire des techniques et de l'environnement du Cnam (CDHTE-Cnam), Société des élèves du CDHTE-Cnam

\section{Édition imprimée}

Date de publication : 1 décembre 2010

Pagination : 269-276

ISBN : 978-2-9530779-5-7

ISSN : 0417-8726

\section{Référence électronique}

François Jarrige, «Se prémunir contre les préjugés ouvriers.

L'économie politique des machines entre l'Angleterre et la France(1800-1850) », Documents pour I'histoire des techniques [En ligne], 19 | 2e semestre 2010, mis en ligne le 21 juin 2011, consulté le 08 septembre 2020. URL : http://journals.openedition.org/dht/1518; DOI : https://doi.org/10.4000/dht. 1518 


\title{
Se prémunir contre les préjugés
}

\section{ouvriers. L'économie politique des \\ machines entre l'Angleterre \\ et la France (1800-1850)}

\author{
François Jarrige \\ Université de Bourgogne \\ Centre Georges Chevrier
}

$P^{o r}$ our l'économiste Joseph Garnier, c'est grâce à « la vulgarisation des premières notions d'économie politique dans les écoles», que les «enfants qui seront un jour ouvriers commenceront à comprendre la véritable nature des choses et seront prémunis contre les préjugés qui les incitent plus tard à haïr les machines, à les briser, ou à compter sur des moyens chimériques ${ }^{1}{ }^{1}$. Aux lendemains des révolutions de 1848 et leur cortège d'émeutes et d'insurrections, l'économie politique entend être une science pacificatrice. Parmi les préjugés qu'elle doit combattre pour préserver l'ordre social, Joseph Garnier met en avant ceux qui ont trait aux machines et aux nouveautés techniques dont la circulation ne cesse de croître au XIX ${ }^{\mathrm{e}}$ siècle.

$\mathrm{Au}$ cours de la première industrialisation, les indus-triels français importent à la fois des machines et la main-d'œuvre pour les faire fonctionner. Les figures de techniciens installés sur le continent au XVIII siècle sont célèbres : John Kay qui émigre avec sa navette volante après un procès perdu, les Milne qui introduisent les water frames, ou William Wilkinson qui est à l'origine de la fabrique de canons d'Indret en 1778. La période de la Restauration et de la Monarchie de Juillet connaît une multiplication de ces échanges de part et d'autre de la Manche ${ }^{2}$. Mais l'arrivée de ces mécaniques suscite de nombreuses oppositions et contestations: ne risquent-elles pas de priver les artisans de leur emploi ? Ne menacent-elles pas l'ordre

1 Joseph Garnier, art. "Machines », dans Charles Coquelin et Gilbert-Urbain Guillaumin dir., Dictionnaire de l'économie politique, 2 vols., Paris, Guillaumin, 1853, vol. 2, cit. p. 122.

2 Michel Cotte éd., Les circulations techniques. En amont de I'innovation: hommes, objets et idées en mouvement, Belfort/ Besançon, UTBM/Presses universitaires franc-comtoises, 2004. social en imposant une industrialisation destructrice sur le modèle anglais? Ne risquent-elles pas de remettre en cause la qualité des produits et donc la force des productions nationales ?

Face à ces inquiétudes qui s'expriment de multiples manières dans la société française, et qui sont sans cesse disqualifiées comme d'absurdes "préjugés », les transferts techniques ont du être associés au transfert des répertoires de justification nécessaires pour acclimater socialement les nouveaux procédés. Cette justification du machinisme est née dans un cadre largement transnational. Elle a pris la forme d'une économie politique industrialiste associant le langage de la science et celui de la réforme sociale. En Angleterre, la précocité industrielle et les menaces que suscitaient les concentrations ouvrières ont poussé très tôt les élites britanniques à développer une pédagogie industrielle pour les foules. L'économie politique, qui s'est constituée en résolvant la "question des machines ${ }^{3}$, a fourni un large répertoire d'arguments aux pédagogues de I'univers industriel. Ces arguments se sont rapidement internationalisés. En France, ils sont importés et retraduits dans les contextes d'agitation politique et sociale, notamment durant la période troublée qui suit la Révolution de Juillet $1830^{4}$.

3 Comme I'a montré Maxine Berg, The machinery question and the making of political economy (1815-1848), Cambridge, Cambridge university press, 1980.

4 Pierre Bourdieu, "Les conditions sociales de la circulation internationale des idées ", Actes de a Recherche en sciences sociales, $n^{\circ} 145,5,2002$, pp. 5-8. 


\section{Les conflits sociaux et l'invention de la pédagogie des machines en Angleterre}

Dès le début du XIX' siècle, I'économie politique expose les lois censées régir l'économie et la société. La question de l'effet des machines est abondamment débattue. Les troubles sociaux qui accompagnent régulièrement les tentatives de mécanisation du travail poussent les élites économiques et politiques à mettre sur pieds une pédagogie industrialiste pour les classes populaires. Peu après les désordres qui affectent le Lancashire en 1779-1780, le magistrat Dorning Rasbotham publie ainsi plusieurs brochures pour montrer aux ouvriers les effets néfastes de leurs actions contre les machines ${ }^{5}$. Quelques mois avant sa mort en 1780, Thomas Bentley, un industriel du textile et un fabricant de porcelaines associé à Josiah Wedgwood, publie à son tour une brochure qui stigmatise «les préjugés contre

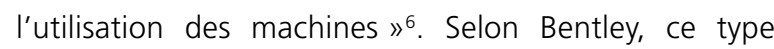
d'erreur fréquent chez les ouvriers risque de leur être fatale, elle procède en général de «l'ignorance et de I'immoralité $»^{7}$. À l'époque du luddisme (1811-1812), les textes se proposant d'expliquer les effets des machines ressurgissent. Dans sa « lettre aux Luddites » publiée en 1816, le radical William Cobbett expose les avantages du machinisme; pour lui, il n'existe pas « d'objection solide » contre les machines puisque ce sont elles qui "distinguent I'homme civilisé du sauvage ${ }^{8}$. Ces efforts pédagogiques ne cessent de croître au cours des années 1820 alors que les bris de machines se poursuivent dans l'industrie textile puis dans le secteur agricole, et que les tisserands à la main engagent des campagnes de pétitions au Parlement pour lutter contre la mécanisation.

5 Thoughts on the use of machines, in the cotton manufacture. Addressed to the working people in that manufacture, and to the poor in general. By a friend of the poor, Manchester, 1780. L'auteur exerça diverses fonctions de magistrat à Manchester et publia des travaux sur I'histoire du Lancashire, on lui attribue également la publication d'une autre brochure sur les machines en 1779: A dissuasive from popular rioting directed against mechanical manufacturing improvements (1779).

6 Thomas Bentley, Letters on the utility and policy of employing machines to shorten labour; occasioned by the late disturbances in Lancashire: to which are added some hints for the further extension and improvement of our woollen trade and manufactures, Londres, 1780.

7 Ibid., p. 2.

8 Leonora Nattrass éd., William Cobbett: selected writings, 6 vols, Pickering and Chatto, 1998, vol. 3: "A Letter to the Luddites (1816)", p. 367. Sur les débats économiques qui accompagnent la fin du luddisme, voir Vincent Bourdeau, François Jarrige et Julien Vincent, Les Luddites, Bris de machines, économie politique et histoire, Paris, Ère édition, 2006, chap. 2.
Benjamin Heywood, un riche philanthrope à l'origine du Mechanics' Institute de Manchester en 1825, dénonce aussi les "hommes ignorants [qui] ont commis I'action honteuse de détruire les métiers mécaniques ». Face à ce type de violence, l'éducation populaire doit montrer que, même «si l'introduction des nouvelles machines remplaçant le travail manuel peut occasionner une détresse temporaire », néanmoins, les métiers qui ont été détruits devaient " permettre une augmentation des salaires et une amélioration des conditions de travail $»^{9}$. Les conférences portant sur cette question sont récurrentes. À Newcastle, deux conférences sont organisées, en mai 1825 puis en mai 1826, pour démontrer «l'utilité des machines pour promouvoir le confort et le bonheur des classes ouvrières dans la société ». À Leeds, en novembre 1830, alors que les émeutes contre les machines se sont renouvelées dans le pays, Edward Baines insiste sur les dangers que ce type de violences représente pour la compétitivité nationale. L'année suivante, en 1831, Birbeck fait une conférence à Londres sur l'avantage de I'emploi des machines ${ }^{10}$.

Face aux agitations et aux revendications sociales menaçantes pour l'ordre social, les auteurs des classes moyennes choisissent donc de diffuser les principes de l'économie politique de la machine. C'est l'une des fonctions de la Société pour la diffusion des connaissances utiles fondée en 1825 et des périodiques comme The Mechanic's magazine ou The Penny magazine, où sont longuement exposés les avantages des machines ${ }^{11}$. Autour de 1830, alors que les émeutes $\mathrm{du}$ "Capitaine Swing » impressionnent vivement les autorités politiques et les philanthropes, de nombreux tracts destinés aux classes populaires exposent les bienfaits du machinisme pour la prospérité du pays ${ }^{12}$. Dans An Adress to the Labourers, on the Subject of Destroying Machinery - un petit texte de huit pages publié en décembre 1830 et vendu un penny - la Société pour la diffusion des connaissances utiles rappelle que

9 Benjamin Heywood, Addresses delivered at the Manchester Mechanics' Institution, London, 1843, pp. 29-31, il s'agit d'un recueil de conférences faites dans les années 1820-1830, cité dans Mabel Tylecote, The Mechanics' Institutes of Lancashire and Yorkshire before 1851, Manchester, Manchester University Press, 1957, p. 132.

10 M. Berg, The machinery question, op. cit., pp. 165-166.

11 Max Goldstrom, «Popular political economy for the British working class reader in the nineteenth century », dans Terry Shinn et Richard Whitley éd., Expository Science: forms and functions of popularization, 1985, pp. 259-273 ; Valérie Gray, "Charles Knight and the Society for the diffusion of the useful knowledge: a special relationship (1827-1846) », Publishing history, 2003 (53), pp. 23-74.

12 Eric Hobsbawm et Georges Rudé, Captain Swing, Londres, Lawrence and Wishart, 1969. 
les machines sont profitables aux classes populaires en permettant l'accroissement de la production et de la consommation. Plus ambitieux, le texte anonyme intitulé Results of Machinery, Namely, Cheap Production and Increased Employment Exhibited: Being an Adress to the Working-Men of the United Kingdom est publié en 1831 dans la série The Working Man's Companion. La même année, de larges extraits en sont également publiés dans le Mechanic's magazine. Pour l'auteur, s'opposer aux machines, c'est s'opposer au meilleur ami du pauvre ${ }^{13}$. La peur sociale qui saisit les élites philanthropiques devant ces évènements violents apparaît dans toute sa clarté dans ces textes : « si la force brutale qui cherche à détruire les machines n'était pas réprimée par le pouvoir de la loi, et si les préjugés imprudents qui désirent leur suppression n'étaient pas conquis par le pouvoir de la raison, alors la gloire et la prospérité de ce pays seraient à jamais anéanties ${ }^{14}$. II en va donc de la grandeur nationale britannique.

Après 1830, les efforts de popularisation de l'économie politique des machines se poursuivent en Grande-Bretagne. Certaines femmes, comme Jane Marcet, qui s'adressaient auparavant aux membres de la bourgeoisie se tournent de plus en plus vers le public ouvrier pour lui enseigner les bienfaits de l'épargne et des machines ${ }^{15}$. Lors des premières éditions de ses « conversations sur l'économie politique », Jane Marcet s'adressait d'abord aux jeunes filles de la classe moyenne : son texte se présentait d'ailleurs comme un dialogue entre une jeune fille de la bourgeoisie nommée Caroline et $\mathrm{Mme} \mathrm{B}^{16}$. Le livre est réédité sept fois entre 1816 et 1839. Dans son texte John Hopkins's Notions of Political Economy, publié en 1833, le désir de s'adresser à un public populaire apparaît plus nettement. Cette fois, elle met en scène un simple labourer qui transmet les principes de base de l'économie politique à travers

13 "The Result of Machinery », Mechanics Magazine, n 387, 8 janvier 1831, p. 323.

14 "The Result of Machinery », Mechanics Magazine, n 389, 22 janvier 1831, p. 357 : « if the brute force which seeks to destroy machinery were not to be put down by the power of the laws, and if the unwise prejudice which desires to repress it could not be conquered by the power of reason - the glory and prosperity of this country would be gone for ever ».

15 Ses textes les plus célèbres sont : Conversations on Chemistry (1806) et Conversations on Political Economy, in which the elements of the science are familiarly explained (1816).

$16 \mathrm{~J}$. R. Shackleton, " Jane Marcet and Harriet Martineau: pioneers of economics education ", History of Education, 1990, vol. 19, n 4, p. 289; Bette Polkinghorn, Jane Marcet. An uncommon woman, Aldermaston, 1993; et Hilda Hollis, "The rhetoric of Jane Marcet's popularizing political economy », Nineteenth century contexts, 2002, vol. 24 (4), pp. 379-396. une série de récits imagés. Dans I'un d'entre eux, John Hopkins tente longuement de convaincre son voisin, un tisserand qui a perdu son emploi à cause d'une machine, des bienfaits des innovations techniques pour la société dans son ensemble.

À la même date, Harriet Martineau, largement influencée par son aînée Jane Marcet, publie une série d'ouvrages de vulgarisation des principes de l'économie politique qui ont un succès considérable ${ }^{17}$. Elle est née en 1802 dans une famille protestante qui avait émigré en Angleterre après la révocation de l'édit de Nantes. En 1827, après avoir lu dans la presse le récit de violences ouvrières, elle écrit une nouvelle sur ce sujet intitulée The rioters où elle met en scène les ouvriers de Manchester se soulevant contre la mécanisation. Devant le succès de ce récit, elle décide de se consacrer à la popularisation des principes économiques et publie d'autres nouvelles qui obtiennent un grand succès. Le deuxième de ses " contes», intitulé "La colline et la vallée », reprend le thème des émeutes ouvrières contre les machines. Dans une région rurale du sud du Pays de Galles, les ouvriers employés dans des forges se révoltent contre des machines qu'ils accusent de prendre leur emploi. Elle élabore un récit très moralisateur où l'ignorance et les préjugés populaires deviennent la source de la ruine. Elle accompagne son récit du résumé des principes économiques que le lecteur doit retenir: "Puisque le capital découle du travail, tout ce qui peut économiser le travail permet l'accroissement du capital. Le machinisme économisant le travail, il permet donc un accroissement du capital. En assistant la croissance du capital, il permet en dernier lieu une augmentation de la demande de travail ${ }^{18}$. Au moyen de ces récits brefs et distrayants, elle diffuse une vision optimiste de l'industrialisation et entend répondre aux inquiétudes qui s'expriment dans certains groupes ${ }^{19}$.

17 Illustration of Political economy, Londres, Charles Fox, 18321833, trad. fr. par B. Maurice : Contes sur l'économie politique, Paris, Charles Gosselin, 8 vol., 1833-1839. Après un grand succès dans les années 1830 , elle tombe dans I'oubli avant de susciter un nouvel intérêt dans l'historiographie anglaise de la seconde moitié du XXe siècle, voir: R. K. Webb, Harriet Martineau. A Radical Victorian, London, Heinemann, 1960 ; Willie Henderson, " Harriet Martineau or 'When political economy was popular' », History of education, 1992, vol. 21, n 4, pp. 383-403.

18 Harriet Martineau, Contes sur l'Economie politique, Paris, Charles Gosselin, 1833, Tome 1er : "La colonie isolée »; «La colline et la vallée »; "Le village et la ferme ».

19 Elaine Freedgood, "Banishing Panic: Harriet Martineau and the Popularization of Political Economy », Victorian Studies, Vol. 39, Number 1, Autumn 1995, pp. 33-53. 


\section{L'économie politique des machines entre l'Angleterre et la France (1800-1850)}

\section{Ure et Babbage, ou la circulation de la science des machines}

En France, l'enseignement de l'économie politique se structure plus lentement qu'en Grande-Bretagne et la question des machines apparaît moins urgente. Pourtant, comme en Angleterre, I'époque révolutionnaire avait vu se multiplier les projets d'une pédagogie industrielle comme ceux formulés par Condorcet ou l'abbé Grégoire. Le premier affirmait que «l'instruction publique est nécessaire pour préparer les nations aux changements que le temps doit amener », en particulier « l'introduction des machines qui, employant moins de bras, force les ouvriers à chercher d'autres occupations ${ }^{20}$. L'Abbé Grégoire justifiait de son côté la création du Conservatoire des arts et métiers, en 1794, par la nécessité de répondre à ceux qui prétendent « que le perfectionnement de l'industrie et la simplification de la main-d'œuvre entraînent des dangers, parce qu'ils ôtent les moyens d'existence à beaucoup d'ouvriers », " la conséquence de cette objection puérile, ajoute-t-il, serait de briser les métiers à bas, les machines à mouliner la soie, et tous les chefs-d'œuvre qu'enfanta I'industrie pour le bonheur de la société» ${ }^{21}$. Le spectre des violences ouvrières nourrit l'ambition vulgarisatrice. Quelques années plus tôt, dans la région rouennaise affectée par des émeutes contre les mécaniques textiles, le bénédictin Maurey réclamait déjà qu' « un cabinet de machines » soit installé auprès de chaque école créée pour qu'on y expose les « inventions les plus utiles, les modèles de machines à filer le coton [...] toutes les machines relatives aux Manufactures ». Grâce à ces «cabinets de machines», concluait-il, "l'ignorance cesseroit, le préjugé, l'entêtement des ouvriers rentreroient dans le néant, on aurait des artistes plutôt que des ouvriers ${ }^{22}$.

Dans la première moitié du siècle, cette volonté répétée de lutter contre les préjugés ouvriers au moyen d'une pédagogie des machines se nourrit des expériences

20 Nicolas de Condorcet, "Nature et objet de l'instruction publique ", dans Cinq mémoires sur l'instruction publique (1791), Paris, Garnier-Flammarion, 1994, p. 24.

21 « Rapport de Grégoire au Conseil des Cinq-cents, demandant I'affectation, au Conservatoire, de la plus grande partie des bâtiments et des terrains de l'ancien prieuré de Saint-Martin des Champs (26 floréal an VI-15 mai 1798) », dans Recueil des lois, décrets, ordonnances, arrêtés, décisions et rapports relatifs à l'origine, à l'institution, à l'organisation et à la direction du Conservatoire national des arts et métiers et à la création des cours publics de cet établissement, Paris, Imprimerie nationale, 1889, pp. 28-40.

22 Jacques-Antoine de Maurey (membre de l'Académie des sciences, belles-lettres et arts de Rouen), Mémoire sur les moyens de propager et de perfectionner les arts mécaniques, Rouen, Gallier, 1791, p. 10.
d'outre-Manche et stimule divers mouvements de traduction. Alors que les agitations sociales et le spectre révolutionnaire ressurgissent après la Révolution de Juillet 1830, le recours à l'Angleterre et à ses expériences pédagogiques devient plus massif ${ }^{23}$. Face aux violences ouvrières, légitimées par certains penseurs traditionalistes préoccupés par les conséquences du modèle de développement industriel britannique ${ }^{24}$, les autorités et les élites libérales importent d'abord les grands traités de la science des machines qui fixent la nouvelle orthodoxie technicienne. En traduisant notamment les ouvrages de Charles Babbage et Andrew Ure, on cherche des arguments qui justifieraient scientifiquement la mécanisation ${ }^{25}$. Babbage et Ure s'affirment alors comme les deux principaux théoriciens de la "science des machines ». Ils montrent l'apport des nouvelles technologies pour l'ordre manufacturier.

Dans son Traité sur l'économie des machines, le mathématicien Babbage expose ainsi, comme le dit son traducteur français Edouard Biot, les « avantages qui résultent de l'emploi illimité des machines comme moyen de production ${ }^{26}$. Dans le chapitre 32 , il se demande si "I'introduction des machines dans un genre d'industrie quelconque a pour effet de diminuer la quantité de main-d'œuvre qui s'y trouve employée ? »27. L'une des principales objections soulevées contre l'usage des machines vient de ce qu' « on leur a reproché de supprimer le travail d'une grande quantité d'ouvriers auparavant

23 La révolution de 1830 replace en effet la question des machines au centre des débats en France, les bris de machines se multiplient après la révolution de Juillet. En province, des machines textiles sont détruites dans le Nord, en Alsace, à Reims ; à Paris, la révolte des typographes contre les premières presses mécaniques frappe les esprits, voir François Jarrige, Au temps des «tueuses de bras». Les bris de machines à l'aube de l'ère industrielle, Rennes, Presses Universitaires de Rennes, 2009 ; et F. Jarrige, «Les ouvriers parisiens et la question des machines au début de la monarchie de Juillet », in Patrick Harismendy (dir), La France des années 1830 et l'esprit de réforme, Rennes, PUR, 2006, pp. 211-222.

24 Comme les traités du baron de Morogue, De l'utilité des machines, de leurs inconvénients et des moyens d'y remédier en assurant l'extension et les progrès de notre agriculture, par M. le Bon de Morogues,... Mémoire présenté à l'Académie royale des sciences en 1831, Paris, Imprimerie royale, 1833, 24 p.

25 Lucette Le Van Lemesle, " Les économistes français et l'usage des modèles étrangers ", Revue d'histoire du XIXe siècle, $n^{\circ} 2$, 2002, pp. 73-86.

26 Charles Babbage, On the economy of machinery and manufacture, 1832 ; trad. fr. : Traité sur l'économie des machines et des manufactures, trad. de l'anglais sur la 3 ème éd par Ed. Biot, Paris, Bachelier, 1833, avertissement du traducteur.

27 Ibid., pp. 439-449. 
employés ». Au moyen de statistiques recueillies dans 65 fabriques de Stockport, Babbage montre au contraire que la mécanisation permet un accroissement de main-d'œuvre employée. La ville de Stockport située au nord-est de Manchester fournissait un exemple particulièrement intéressant : située au cœur de la grande région cotonnière du Lancashire, l'industrialisation s'y était développée rapidement en engendrant une forte croissance démographique et de multiples contestations de la part de la main-d'oeuvre ${ }^{28}$.

Andrew Ure, chimiste et pédagogue célèbre, "le Pindare de la fabrique » selon le mot de Marx ${ }^{29}$, va plus loin encore dans sa " philosophie des manufactures ${ }^{30}$. II y décrit longuement les immenses possibilités de contrôle permis par la machine et le système de la fabrique; il expose les principes généraux à partir desquels l'industrie pourrait être conduite par des machines autonomes. Babbage et Ure sont les rares auteurs à considérer la mécanisation comme le phénomène central de l'économie industrielle naissante ${ }^{31}$. Au cours des années 1830, les analyses mathématiques et statistiques sont mises au service de l'économie politique pour démontrer les bienfaits des machines sur la main-d'œuvre. John Edward Tozer, membre du groupe d'économie mathématique qui entourait William Whewell à Cambridge, publie ainsi un article qui démontre, au moyen d'outils mathématiques, l'erreur de Ricardo dans son chapitre sur les machines ${ }^{32}$.

28 Sur l'industrialisation de Stockport, voir Robert Glen, Urban workers in the early Industrial Revolution, Londres, Croom Helm, 1984. La population du district de Stockport était passée de 12000 habitants au milieu du XVIIIle siècle, à près de 49000 en 1811 et 82000 en 1831. Par ailleurs, la ville de Stockport avait été au cœur du soulèvement luddite dans le Lancashire en 1812 (chap. 8: "The Year of the Luddites », pp. 167-193), dans les années 1820 , les tisserands à la main continuaient d'entretenir une vive agitation. Le choix de cette ville pour démontrer les avantages de la mécanisation ne relevait donc en rien du hasard. 29 Karl Marx, Fuvres. Economie, I: Le Capital, [1867], Paris, Gallimard, coll. « La pléiade », 1963, p. 951.

30 Andrew Ure, The philosophy of manufacture, 1835, trad. fr. : Philosophie des manufactures ou économie industrielle de la fabrication du coton, 2 vols., Paris, L. Mathias, 1836, vol. 2, $3^{\text {ème }}$ partie, chap. 1, p. 17.

31 Andrew Zimmerman, "The Ideology of the machine and the spirit of the factory: remark on Babbage and Ure », Cultural critique, no 37, 1997, pp. 5-29.

32 John Edward Tozer, "Mathematical investigation of the effect of machinery on the wealth of a community in which it is employed and on the fund for the payment of wages ", Transactions of the Cambridge Philosophical Society, vol. VI, part III,1838, pp. 507-22, I'apport de Tozer au débat contemporain sur les effets des machines est étudié par Christian Gehrke, "Tozer on machinery », The European Journal of the History of
Les traductions des textes de Babbage et de Ure constituent donc des étapes importantes dans la constitution d'idéologies techniques au service du développement industriel.

Le Traité sur l'économie des machines et des manufactures, publié en anglais en 1832, est traduit en français dès 1833 par Edouard Biot, un ingénieur polytechnicien qui était aussi membre de la Société d'encouragement pour I'industrie nationale. Dans la préface qu'il rédige, puis dans un article du Journal des Savants où il présente l'étude de Babbage, Biot assure qu'il voit dans le livre un outil utile pour répondre aux émeutes ouvrières. Babbage démontre en effet qu'en permettant d'accroître la consommation grâce à la diminution des prix, les machines augmentent en définitive la production et donc l'emploi. Ainsi, « l'examen des effets généraux des machines, et sa solution serait d'une grande utilité pour rassurer la classe ouvrière sur sa position, dans l'état toujours progressif du perfectionnement des agents mécaniques, et de leurs applications à toute espèce de production $»^{33}$. Par ailleurs, souligne-t-il, « les coalitions que forment les ouvriers pour obtenir une augmentation de salaire, ou pour s'opposer à l'introduction d'une nouvelle machine, ont toujours un résultat funeste pour eux, parce que la fabrication se déplace alors, va s'établir sur des points éloignés de cette sorte de fermentation populaire ».

Dès l'année suivante, une seconde traduction française du livre de Babbage paraît sous le titre de Science économique des manufactures ${ }^{34}$. Cette fois, le traducteur, nommé Isoard, est un haut fonctionnaire du ministère du Commerce. La visée pédagogique apparaît nettement puisqu'il avait reçu une récompense de 1500 francs pris sur le fonds des brevets d'invention à titre « d'indemnité pour travaux et traductions d'ouvrages anglais utiles à I'industrie ${ }^{35}$. Après un voyage en Angleterre, le ministre du Commerce, frappé du prodigieux succès de l'ouvrage qui en était déjà à sa troisième édition, décide de le faire traduire pour convaincre les fabricants de se lancer dans la modernisation de leurs procédés. Babbage est alors un auteur majeur qui exerce une grande influence en Angleterre. Son originalité vient de ce qu'il adopte des conceptions très larges, il ne s'agit pas d'une simple compilation d'observations dispersées mais d'une analyse globale du système manufacturier de production. En France, son influence fut plus limitée qu'en Grande-

Economic Thought, 7, 4, 2000, p. 485-506.

33 Edouard Biot, " On the Economy of machinery... », Journal des savants, 1834, pp. 12, 15-16

34 Science économique des manufactures, Paris, Dondey Dupré, 1834. 35 Archives nationales (Paris) : F/12/12368: Récompense à Isoard, Paris, 16 octobre 1833. 


\section{L'économie politique des machines entre l'Angleterre et la France (1800-1850)}

Bretagne, mais sa traduction rapide témoigne néanmoins des vifs débats sur les machines qui agitent les débuts de la monarchie de Juillet.

L'ouvrage d'Andrew Ure, la Philosophie des manufactures, publié à Londres en 1835 est traduit dès 1836. II pose les bases d'une utopie capitaliste du processus de production envisagé sans le travail ${ }^{36}$. La manufacture permet au capitaliste d'exercer un contrôle complet sur la production. Le projet de Ure, fondé sur son expérience personnelle, est purement pédagogique. Son étude est un long exposé des principes généraux par lequel l'industrie pourrait être conduite par des machines autonomes. Ces textes ont exercé une grande influence dans les milieux industriels, ils ont contribué à rendre familier l'interconnexion entre la technologie et I'organisation de la production. Même s'ils n'étaient pas directement diffusés dans le monde ouvrier, ils étaient trop théoriques et s'adressaient d'abord aux industriels et aux classes moyennes, ils ont servi néanmoins de fondement à la nouvelle science des machines qui justifiait la généralisation de leur emploi. Pour ces auteurs, les violences populaires sont le résultat de l'ignorance des vrais principes de l'économie politique. Pour restaurer I'ordre, il suffit donc de diffuser le plus largement possible la pédagogie des machines.

\section{L'importation de la pédagogie anglaise des machines en France dans les années 1830}

«Comment faire pour que l'ouvrier bénisse les machines ? » demande le fabricant Arlès-Dufour en $1841^{37}$. Au début des années 1830, la solution semblait résider dans la diffusion des Lumières venues d'outre-Manche. Dès 1831, Emile de Girardin avait ouvert la voie en publiant le Journal des connaissances utiles directement traduit de la Society for the diffusion of useful knowledge. Ce périodique avait connu un extraordinaire succès au début de la monarchie de Juillet avec 40000 abonnés en février 1832, 114000 en décembre 1832 et 125000 , à son apogée en $1833^{38}$. La Société pour I'instruction élémentaire a également joué un rôle actif dans ce mouvement. Dès le mois d'août 1830, elle propose un prix de 500 francs « en faveur du meilleur écrit populaire destiné à prouver l'heureuse influence des machines sur l'aisance de toutes les classes de citoyens, et particulièrement sur celle de la classe ouvrière, ainsi

36 Steve Edwards, "Factory and fantasy in Andrew Ure", Journal of design history, 2001, n 14, pp. 17-34.

37 Cité dans La Ruche populaire, novembre 1841, p. 32.

38 Pierre Albert, « Le Journal des connaissances utiles de Girardin (1831-1836) ou la première réussite de la presse à bon marché », Revue du Nord, 1984 que sur le taux des salaires ${ }^{39}$. Répondant par avance aux objections de certains métiers comme les typographes qui venaient de se soulever contre les presses mécaniques, la société précise que «l'auteur s'attachera à démontrer que les machines sont utiles, même aux ouvriers de la profession dans laquelle elles sont employées ». L'un des 27 mémoires reçus provient significativement de Manchester, où il existait une longue tradition de textes de vulgarisation de ce type.

À côté des initiatives privées, les pouvoirs publics jouent également un rôle difficile à évaluer. En août 1831, dans un long rapport au roi, le comte de Montalivet alors ministre de l'instruction publique et des Cultes, propose la création d'une commission qui serait chargée de l'examen des ouvrages scolaires et populaires édités en Europe occidentale (plus de 1500 ont déjà été recensés, écrit-il). Les meilleurs seraient traduits et diffusés dans les écoles partout en France. « Il importe, écrit-il ensuite, que des explications simples et claires soient données sur les merveilles de la nature, sur les phénomènes qui font naître et entretiennent la plupart des préjugés populaires, sur les procédés des arts utiles et de l'agriculture $»^{40}$.

La Société d'encouragement pour l'industrie nationale, à la pointe du soutien aux innovations, intervient également en faisant traduire, dès 1833, le traité sur I'utilité des machines diffusé en Angleterre en 1831 par la Society of useful knowledge ${ }^{41}$. Dans un rapport de janvier 1834, le baron Séguier propose que ce texte soit diffusé par la Société le plus largement possible :

« la lecture d'un tel ouvrage, sa propagation, sont de nature à déraciner cette foule de préjugés que rencontrent sans cesse toutes les industries naissantes, et nous croyons qu'il entre dans la noble mission dont notre Société s'est chargée, de coopérer, autant qu'il est en elle, à le répandre. Nous avons donc l'honneur [...] d'inscrire ce livre au nombre de ceux qui doivent être distribués aux contre-maîtres $»^{42}$.

39 Bulletin de la société pour l'instruction élémentaire, $n^{\circ} 19$, juillet 1830, p. 128 : «Programme d'un prix de 500 francs ». 40 Rapport publié dans le Moniteur et reproduit dans Les Bibliothèques populaires, scolaires et pédagogiques, documents législatifs et administratifs, recueillis et mis en ordre par B. Subercaze, Paris, P. Dupont, 1892, p. 3-5; voir aussi Noël Richter, La lecture et ses institutions (1700-1918), Editions Plein chant, bibliothèque de l'université du Maine, 1987, pp. 138-139. 41 Des machines et de leurs résultats, trad. de l'anglais par M. Lhuillier de l'Etang, membre de la société d'encouragement pour l'industrie nationale, Paris, Paulin, 1833.

42 «Rapport fait par M. le baron Séguier, sur un ouvrage intitulé : Des machines et de leurs résultats», Bulletin de la Société d'encouragement pour l'industrie nationale, $33^{\text {ème }}$ année, 
Les contremaîtres deviennent ainsi des intercesseurs chargés de diffuser les discours favorables aux machines dans le quotidien des ateliers.

Les expériences de vulgarisation de l'économie politique qui s'étaient développées en Grande-Bretagne retiennent de plus en plus l'attention en France. Les périodiques dédiés à la traduction des textes anglais, comme La revue britannique ou L'Écho britannique, contribuent à la diffusion d'une interprétation optimiste de la mécanisation ${ }^{43}$. Les récits populaires de Jane Marcet et Harriet Martineau sont traduits rapidement après leur publication en anglais. En 1834, parait ainsi la traduction des Conversations sur l'économie politique et des Notions de John Hopkins sur l'économie politique. Jane Marcet était liée au réseau des savants genevois, elle vécut plusieurs années en Suisse et c'est l'éditeur Cherbuliez qui publie les traductions de ses livres. Dans ces textes, il s'agit moins d'enseigner et d'informer que de prêcher et de persuader ; l'enseignement principal des récits de Marcet est que le bien-être des riches est profitable aux classes populaires. En accroissant les capacités productives des industriels c'est toute la société qui devait bénéficier des machines. Mais cette idée apparaît en contradiction avec les expériences propres du monde ouvrier de l'époque; la visée moralisatrice et l'absence d'ambition réformatrice de ces textes alors qu'émerge, à la même période, un discours socialiste qui replace les machines dans l'idéal de l'association, peut expliquer leur manque de succès en France.

Les ouvrages de Harriet Martineau, quant à eux, sont traduits par B. Maurice chez Gosselin à partir de 1833. « Un libraire de province, explique-t-elle à son traducteur français, me demanda de composer pour lui quelque petit ouvrage dans le genre narratif ; je pensais que je pourrais joindre l'utile à l'agréable, si, ayant le choix d'un sujet, je parvenais à démontrer la sottise de la populace de Manchester, qui venait précisément de détruire les machines, au grand détriment des manufactures, dont son pain dépendait ${ }^{44}$. La traduction de l'ouvrage de Martineau reçoit d'ailleurs le soutien des autorités politiques. Molinari, qui republie les « contes » en 1880, assure que Louis-Philippe fit distribuer des exemplaires du livre aux membres de sa famille, et Guizot en aurait fait acheter « un grand nombre d'exemplaires » sur les fonds du ministère de I'Instruction publique pour les distribuer gratuitement dans les écoles primaires ${ }^{45}$.

janvier 1834, p. 43-44.

43 «Les machines. Effet de leur introduction pour la classe ouvrière », Revue britannique, $3^{\text {ème }}$ série, XVI, juillet-août 1835, p. 77.

44 « Lettre de Miss Harriet Martineau à M. B. Maurice », Contes sur l'économie politique, op. cit.

45 Gustave de Molinari, " Miss Harriet Martineau », Journal des
Les attaques ouvrières contre les machines sont évoquées dans ces textes. Martineau met en scène les ouvriers et leurs croyances :

«Les ouvriers croyaient trop fermement que les perfectionnements dans les machines étaient la cause de leurs malheurs, et non la conséquence des remèdes qu'on y voulait apporter, pour que des explications superficielles, ou même la conviction de quelques uns $d^{\prime}$ entre eux suffisent à détruire leur prévention $»^{46}$.

Pourtant, c'est bien à l'énoncé de ces explications que se livre l'auteur. Ces efforts de popularisation retiennent I'attention des réformateurs français. Dans la Revue Encyclopédique par exemple, Adélaïde de Montgolfier, ellemême auteur d'une biographie de Sismondi et traductrice de romans anglais, publie une longue étude consacrée aux « tentatives pour populariser l'économie politique en Angleterre ${ }^{47}$. Elle y présente les contes d'Harriet Martineau, mais aussi les ouvrages de la « Working Man's Companion » et une brochure du théologien Thomas Chalmer. Cette littérature de vulgarisation est accueillie avec ambivalence en France où le langage économique ne possède pas le même poids dans l'espace public. Ainsi, Adélaïde Montgolfier reconnaît certes que « l'émeute, les coalitions pour ruiner les fabricants, l'incendie des grains, le brisement des mécaniques, l'assassinat des douaniers sont des maux pour tous », mais « ces secousses ne sont pas les suites d'un plan de conduite raisonné qu'un autre raisonnement mieux fondé puisse changer, ce sont des cris d'angoisse » ; ainsi mieux vaudrait " guérir les maux » de l'ouvrier que « de le convaincre qu'il a tort de crier $»^{48}$. Elle remet en cause le principe même de la popularisation de l'économie politique au profit d'un appel à la réforme sociale et à l'association.

Durant la première industrialisation, les discours de justification et la popularisation des bienfaits du machinisme circulent abondamment entre la France et I'Angleterre. De part et d'autre de la Manche, selon des tempo variables, la presse à bon marché, l'enseignement technique, les expositions et les conférences, la littérature édifiante et les ouvrages de vulgarisation, ont contribué à l'imposition d'un capitalisme industriel qui n'allait pas de soi. Cette vulgarisation a emprunté le langage de l'économie politique, elle a contribué à l'émergence progressive d'un consensus sur le nouvel ordre industriel.

économistes, juillet-sept. 1880, pp. 54-65, p. 60.

46 Harriet Martineau, Contes sur l'Economie politique, op. cit., Tome 1er, chap. 7, p. 231.

47 Adelaïde de Montgolfier, «Tentatives pour populariser I'économie politique en Angleterre », Revue Encyclopédique, 1832, pp. 650-663.

48 lbid., p. 662. 


\section{L'économie politique des machines entre l'Angleterre et la France (1800-1850)}

En luttant contre « les préjugés ouvriers », elle a façonné les idéologies techniciennes du monde contemporain. En Angleterre, cette pédagogie industrialiste apparaît très tôt. Selon Michel Chevalier, c'est d'ailleurs cette précocité qui explique l'absence de Révolution outre-Manche. Pour lui, « il n'est pas douteux que si l'enseignement de l'économie politique avait été plus répandu, s'il eût été mis à la portée du vulgaire avant le mois de février 1848, les doctrines qui firent de si grands ravages, n'auraient pas trouvé d'écho » en France ${ }^{49}$. En dépit de

49 Michel Chevalier, «L'économie politique et le socialisme. Discours prononcé au Collège de France, le 28 février, pour son caractère simpliste, cette explication nous renseigne sur l'ampleur des attentes que suscitait la popularisation de l'économie politique au milieu du siècle. Par la suite, I'institutionnalisation progressive de l'enseignement économique et le champ des expérimentations vulgarisatrices continuent de s'étendre parallèlement au désir de restauration de l'ordre social et d'affirmation du monde industrie ${ }^{50}$.

l'ouverture du cours d'économie politique », Journal des économistes, $n^{\circ}$ 93, mars 1849, pp. 25-26.

50 Lucette Le Van-Lemesle, Le Juste ou le Riche. L'enseignement de l'économie politique en France, 1815-1950, Paris, CHEFF, 2004. 